\title{
Erratum
}

\section{Direct Etherification of Alkyl Halides by Sodium Hydride in the Presence of $N, N$-Dimethylformamide}

Cheng Hua Jin, Ho Young Lee, Sang Hwi Lee, In Su Kim, Young Hoon Jung* Synlett 2007, 2695-2698.

The advanced online publication (e-First) contained three errors that have been corrected for both the print and the current online versions (see below).

(1) The last sentence of the second paragraph on page 2 has been deleted from the e-First version.

(2) In the third paragraph on page 2, the author refers to the homo- and cross-etherification of various alkyl bromides (3-7); an incorrect range of compound numbers had been given here.

(3) On page 3, the last sentence before Scheme 1 was incorrect and mentioned 'the formation of ether $\mathbf{5}$ ' instead of 'the formation of ethers.' 